\title{
Long noncoding RNA HOTAIR promotes the proliferation and metastasis of osteosarcoma cells through the AKT/mTOR signaling pathway
}

\author{
ENQI LI ${ }^{1}$, ZHE ZHAO $^{2}$, BAOTONG MA ${ }^{1}$ and JINLI ZHANG ${ }^{1}$ \\ Departments of ${ }^{1}$ Orthopaedic Trauma and ${ }^{2}$ Surgery of Traditional Chinese Medicine, \\ Tianjin Hospital, Tianjin 300211, P.R. China
}

Received October 6, 2016; Accepted July 27, 2017

DOI: $10.3892 /$ etm.2017.5248

\begin{abstract}
It has been proven that long non-coding (lnc)RNAs serve an important role in the tumorigenesis and development of several types of human malignancy. Previous studies have demonstrated that the IncRNA Hox transcript antisense intergenic RNA (HOTAIR) is involved in the development various types of cancer, including osteosarcoma (OS). However, the underlying mechanisms by which it has an affect are still largely unknown. In the present study, it was observed that the expression of HOTAIR was significantly upregulated in OS tissues compared to matched adjacent normal tissues, using reverse transcription-quantitative polymerase chain reaction analysis. HOTAIR was silenced using specific small interfering RNA (siRNA/siR), siR-HOTAIR, in order to investigate its role in regulating OS cell proliferation, apoptosis, migration and invasion. siR-HOTAIR inhibited the proliferation of MG-63 cells due to the induction of G1 phase arrest. In addition, the results of in vitro assays demonstrated that the suppression of HOTAIR in MG-63 OS cells significantly reduced migration and invasion. The silencing of HOTAIR also significantly decreased the expression of matrix metalloproteinase (MMP) 2 and MMP9, but increased E-cadherin expression through regulating the RAC $\alpha$ serine/threonine protein kinase-mammalian target of rapamycin signaling pathway. The results indicated that siR-HOTAIR may be a potential OS therapy.
\end{abstract}

\section{Introduction}

Osteosarcoma (OS) is the most common type of primary bone cancer in children and adolescents (1). Despite medical

Correspondence to: Dr Jinli Zhang, Department of Orthopaedic Trauma, Tianjin Hospital, 406 South Jiefang Road, Tianjin 300211, P.R. China

E-mail: zhangjinlitjyy@126.com

Key words: long non-coding RNAs, Hox transcript antisense intergenic RNA, osteosarcoma, proliferation, metastasis advances and the development of novel treatments, the 5-year survival rate $(65 \%)$ for patients with OS remains poor (2) and the underlying mechanisms of bone carcinogenesis have remained elusive. Therefore, research into these underlying molecular mechanisms is urgently required.

Long non-coding $(\operatorname{lnc})$ RNAs are evolutionarily conserved sections of RNA that are $>200$ nucleotides in length. Numerous lncRNAs do have known biological functions, such as the transcriptional and translational regulation of protein coding sequences 2 (3). Recent studies have demonstrated that lncRNAs serve a key role within diverse biological processes, including cell cycle progression, differentiation, proliferation, apoptosis and tumorigenesis, by regulating gene expression at the transcriptional and post-transcriptional stages $(4,5)$. The urothelial cancer associated 1 lncRNA is highly expressed in bladder cancer samples and its elevated expression has been correlated with an increased rate of tumor recurrence (6). Metastasis associated lung adenocarcinoma transcript 1 (MALAT1) lncRNA exhibits abnormal expression in numerous types of human carcinoma, including colon, lung, breast and ovarian cancer (7-10). It has been reported that MALAT1 could be a novel biomarker for predicting tumor recurrence after liver transplantation (11). Hox transcript antisense intergenic RNA (HOTAIR) lncRNA is known to bind directly to the polycomb repressive complex 2 (PRC2), and cause silencing of the HOXD antisense growth-associated lncRNA gene (12). Previous studies have reported that HOTAIR is involved in the regulation of tumor invasion and metastasis $(13,14)$. In breast cancer, the overexpression of HOTAIR significantly increases tumor invasion and promotes cancer metastasis by altering the methylation of histone H3 lysine 27 (15). HOTAIR was also identified to be significantly upregulated in ovarian cancer, but knockdown of HOTAIR was revealed to repress cell invasion and the viability of ovarian cancer cells by controlling the expression of Ras-related protein Rab-22A (16). Unlike in other types of cancer, the underlying mechanism by which lncRNA HOTAIR affects the development of OS remains unknown.

In the present study it was demonstrated that HOTAIR was overexpressed in OS samples, and that the silencing of HOTAIR inhibited the growth and invasiveness of MG-63 OS cells. These results suggest that lncRNA HOTAIR is involved 
in the progression of OS and may serve an oncogenic role in promoting the malignancy of OS cells.

\section{Materials and methods}

Patient samples. A total of 60 samples of OS tissues and adjacent non-tumor tissues were obtained from patients who underwent surgery at Tianjin Hospital between February 2011 and January 2015. Two pathologists independently evaluated the histological diagnosis and differentiation of the tissue samples, according to the World Health Organization classification system (5). All of the tissue samples collected were immediately snap-frozen in liquid nitrogen and stored at $-80^{\circ} \mathrm{C}$ until required. The present study was approved by the Research Ethics Committee of Tianjin Hospital (Tianjin, China), and informed consent was obtained from all patients. The clinicopathological characteristics of the patients are provided in Table I; a low expression of HOTAIR was considered to be $<1.2$ times the normal level and a high expression of HOTAIR was considered to be $>1.2$ times the normal level.

Cell culture. The OS cell line MG-63 was purchased from the American Type Culture Collection (Manassas, VA, USA). Cells were maintained in Dulbecco's modified Eagle's medium (DMEM) supplemented with $10 \%$ fetal bovine serum (FBS; Gibco; Thermo Fisher Scientific, Inc., Waltham, MA, USA), $100 \mathrm{U} / \mathrm{ml}$ penicillin and $100 \mathrm{mg} / \mathrm{ml}$ streptomycin in a humidified environment at $37^{\circ} \mathrm{C}$ with $5 \% \mathrm{CO}_{2}$.

RNA interference and transfection. Specific small interfering RNA (siRNA/siR) targeting HOTAIR mRNA (siR-HOTAIR1, 3'-GAACGGGAGUACAGAGAGAUU-5'; siR-HOTAIR2, 3'-CCACAUGAACGCCCAGAGAUU-5') and negative control with scrambled sequence (siR-NC) were synthesized by Guangzhou RiboBio Co., Ltd. (Guangzhou, China), and transfected using Invitrogen Lipofectamine ${ }^{\circledR} 2000$ (Thermo Fisher Scientific, Inc.) according to the manufacturer's protocol. A total of $3 \times 10^{5} \mathrm{MG}-63$ cells were seeded into 6-well plates and divided into four groups as follows: Mock group, cells without transfection; siR-NC group, cells transfected with the negative control siRNA; siR-HOTAIR1 group, cells transfected with siR-HOTAIR1; and siR-HOTAIR2 group, cells transfected with siR-HOTAIR2. After $48 \mathrm{~h}$ the effects of gene silencing were measured using reverse transcription-quantitative polymerase chain reaction (RT-qPCR) analysis. HOTAIR was silenced in order to explore the association between the expression of HOTAIR and the behavior of MG-63 cells.

Cell proliferation. The proliferation of MG-63 cells was measured using a Cell Counting Kit (CCK)-8 assay (Dojindo Molecular Technologies, Inc., Kumamoto, Japan) according to the manufacturer's protocol, after siRNA transfection for $0,12,24$ and $48 \mathrm{~h}$. A total of $3 \times 10^{3} \mathrm{MG}-63$ cells were seeded in 96-well plates and $10 \mu \mathrm{l} \mathrm{CCK-8} \mathrm{was} \mathrm{added} \mathrm{and} \mathrm{the} \mathrm{samples}$ were incubated for $4 \mathrm{~h}$ at $37^{\circ} \mathrm{C}$. Subsequently, the OD value was measured at $450 \mathrm{~nm}$ using a Bio-Rad 3550 microplate reader (Bio-Rad Laboratories, Hercules, CA, USA).

Cell cycle analysis. MG-63 cells $\left(3 \times 10^{3}\right)$ were seeded in 6-well plates. After digestion and centrifugation, the plates were fixed in $70 \%$ ethanol at $4^{\circ} \mathrm{C}$ overnight and then washed three times in PBS. A total of $50 \mu \mathrm{g} / \mathrm{ml}$ propidium iodide (PI; Sigma-Aldrich; Merck KGaA, Darmstadt, Germany) was added and the plates were incubated for $30 \mathrm{~min}$ at room temperature in the dark. Measurement of the cell cycle was then performed using a BD FACSCalibur ${ }^{\mathrm{TM}}$ flow cytometer (BD Biosciences, Franklin Lakes, NJ, USA) and analysis was performed using ModFit LT software (Verity Software House, Topsham, ME, USA).

Cell apoptosis analysis. Cell apoptosis was measured using an Annexin V-FITC/PI Apoptosis Detection kit (Beyotime Institute of Biotechnology, Haimen, China) according to the manufacturer's protocol. Briefly, cells $\left(3 \times 10^{5}\right)$ were cultured in 6-well plates, trypsinized $48 \mathrm{~h}$ after transfection and then washed with cold PBS. Cells were then stained by adding the Annexin V reaction mixture consisting of $10 \mu 1$ Annexin V and $5 \mu \mathrm{l} \mathrm{PI}$, and were incubated at room temperature for 15 min in the dark. The stained cells were measured using a BD FACSCalibur according to the manufacturer's protocol and analysis was performed using FlowJo 6 software (FlowJo, LLC, Ashland, OR USA).

$R T-q P C R$ analysis. Total RNA was extracted using TRIzol (Invitrogen; Thermo Fisher Scientific, Inc.) from MG-63 OS cells and tissue samples. RNA Lysis Buffer (RLA) was provide by Promega Corporation (Madison, WI, USA). Complementary (c)DNA was synthesized using a cDNA Synthesis kit (Takara Biotechnology Co., Ltd., Dalian, China) in accordance with the manufacturer's protocol. Then, a SYBR ${ }^{\circledR}$ Premix Ex Taq ${ }^{\mathrm{TM}}$ II kit (Takara Biotechnology Co., Ltd.) was used following the manufacturer's protocol to amplify the cDNA. The PCR condition s were as follows: Denaturation at $94^{\circ} \mathrm{C}$ for $3 \mathrm{~min}$, followed by 30 cycles at $94^{\circ} \mathrm{C}$ for $30 \mathrm{sec}, 55^{\circ} \mathrm{C}$ for $30 \mathrm{sec}$ and a final elongation at $72^{\circ} \mathrm{C}$ for $60 \mathrm{sec}$, then $72^{\circ} \mathrm{C}$ for $10 \mathrm{~min}$. The expression of GAPDH was used as a reference to normalize the amount of IncRNA or mRNA in each sample. The results were quantified using the $2^{-\Delta \Delta \mathrm{Cq}}$ method (17). The PCR primers used were as follows: HOTAIR forward, 5'-GGTAGAAAA AGCAACCACGAAGC-3' and reverse, 5'-ACATAAACCTCT GTCTGTGAGTGCC-3'; G/ $/$ S-specific cyclin D1 (cyclin D1) forward, 5'-TCTCCAAAATGCCAGAGGCGAGGAAGTTG TTGGGGCTCCT; cyclin E forward, 5'-GTGGCTCCGACC TTTCAGTC-3' and reverse, 5'-CACAGTCTTGTCAATCTT GGCA-3'; cyclin-dependent kinase (CDK)-4 forward, 5'-TGC CAATTGCATCGTTCACCGAG-3' and reverse, 5'-TGCCCA ACTGGTCGGCTTCA-3'; CDK2 forward, 5'-GCCTAATCT CACCCTCTCC-3' and reverse, 5'-CCCTTTCACCCCTGT ATTCC-3'; p21 forward, 5'-GTCCTGGATGTTGACTGC CTTGA-3' and reverse, 5'-GTCCAGCAAATCCAAGCTGTC TC-3'; p27 forward, 5'-CATTAACCCACCGGAGCTGTT TAC-3' and reverse, 5'-GGTTAGCGGAGCAGTGTCCA-3'; matrix metalloproteinase (MMP)2 forward, 5'-GTGGATGAT GCCTTTGCTC-3' and reverse, 5'-CAGGAGTCCGTCCTT ACC-3'; MMP9 forward, 5'-AATCTCTTCTAGAGACTG GGAAGGAG-3' and reverse, 5'-AGCTGATTGACTAAA GTAGCTGGA-3'; epithelial (E)-cadherin forward, 5'-CGG GAATGCAGTTGAGGATC-3' and reverse, 5'-AGGATGGTG TAAGCGATGGC-3'; neural (N)-cadherin forward, 5'-ACA GTGGCCACCTACAAAGG-3' and reverse, 5'-CCGAGAT GGGGTTGATAATG-3'; and CD44 forward, 5'-TCCCAG 
Table I. LncRNA HOTAIR expression and the clinicopathological features of the patients with OS.

\begin{tabular}{|c|c|c|c|c|}
\hline \multirow[b]{2}{*}{ Variable } & \multirow[b]{2}{*}{ No. of patients $(\%)$} & \multicolumn{2}{|c|}{ HOTAIR expression (no. of patients) } & \multirow[b]{2}{*}{ P-value } \\
\hline & & Low $(\%)$ & High (\%) & \\
\hline Age (years) & & & & 0.795 \\
\hline$<20$ & $34(100)$ & $16(47)$ & $18(53)$ & \\
\hline$\geq 20$ & $26(100)$ & $14(54)$ & $12(46)$ & \\
\hline Gender & & & & 0.599 \\
\hline Male & $38(100)$ & $22(58)$ & $16(42)$ & \\
\hline Female & $22(100)$ & $11(50)$ & $11(50)$ & \\
\hline Tumor size (cm) & & & & 1.000 \\
\hline$\geq 10$ & $20(100)$ & $10(50)$ & $10(50)$ & \\
\hline$<10$ & $40(100)$ & $19(48)$ & $21(52)$ & \\
\hline Tumor grade & & & & 0.035 \\
\hline I & $9(100)$ & $7(78)$ & $2(22)$ & \\
\hline II & $15(100)$ & $6(40)$ & $9(60)$ & \\
\hline III & $36(100)$ & $11(31)$ & $25(69)$ & \\
\hline Distant metastasis & & & & 0.007 \\
\hline Absent & 37 (100) & $27(73)$ & $10(27)$ & \\
\hline Present & $23(100)$ & $8(35)$ & $15(65)$ & \\
\hline
\end{tabular}

Tumor grading was defined according to the World Health Organization classification system (30). HOTAIR, Hox transcript antisense intergenic RNA.

ACGAAGACAGTCCCTGGAT-3' and reverse, 5'-CACTGG GGTGGAATGTGTCTTGGTC-3'. GAPDH was the control for the PCR assay, with a forward primer sequence of 5'-TGA AGGTCGGAGTCAACGGATTTGGT-3' and reverse primer sequence of 5'-CATGTGGGCCATGAGGTCCACCAC-3'.

Western blot analysis. MG-63 cells were transfected with siR-HOTAIR1 or siR-NC for $48 \mathrm{~h}$, and the proteins were extracted using a radioimmunoprecipitation assay buffer (Beyotime Institute of Biotechnology, Shanghai, China). The Bradford assay was used to determine the protein concentration. A total of $50 \mu \mathrm{g}$ proteins were separated by $10 \%$ SDS-PAGE (Beyotime Institute of Biotechnology) and transferred to polyvinylidene difluoride membranes. The membranes were blocked with $5 \%$ non-fat milk at $4^{\circ} \mathrm{C}$ overnight and then incubated at $4^{\circ} \mathrm{C}$ overnight with rabbit or mouse antibodies specific for the following human proteins: CD44 (1:1,500, sc-65265), CDK4 (1:1,500, sc-23896), MMP2 (1:1,500, sc-13594) and MMP9 (1:1,500, sc-21733) (all from Santa Cruz Biotechnology, Inc., Dallas, TX, USA), cyclin D1 (1:1,500, \#2926), cyclin E (1:1,500, \#2925), p27 (1:1,500, \#3686) (all from Cell Signaling Technology, Inc., Danvers, MA, USA). Antibodies against phosphoinositide 3-kinase (PI3K, 1:1,500, \#4255), RAC $\alpha$ serine/threonine-specific protein kinase (AKT, 1:1,500,\#4060), mammalian target of rapamycin (mTOR, 1:1,500,\#2983), phosphorylated (p)-PI3K (tyr458, 1:1,000, \#4228S), p-AKT (ser473, 1:1,000, \#4060) and p-mTOR (ser2448, 1:1,000, \#5536) were purchased from Cell Signaling Technology, Inc. Mouse anti-human GAPDH antibodies (1:3,000, G8795), which were used as a control, were purchased from Sigma-Aldrich (Merck KGaA). The next day the membranes were probed with secondary anti-rabbit IgG HRP-linked antibody (1:5,000; \#7074) or anti-mouse IgG HRP-linked antibody (1:5,000; \#4408) (both from Cell Signaling Technology, Inc.). After washing three times in TBS-Tween 20, the membranes were incubated with the corresponding secondary antibodies for $1 \mathrm{~h}$ and then detected by an enhanced Pierce chemiluminescence system (ECL; Thermo Fisher Scientific, Inc.).

Cell migration and invasion assay. Cell migration and invasion were assayed using a Transwell invasion assay (invasion Transwell chambers; EMD Millipore, Billerica, MA, USA) with and without Matrigel ${ }^{\mathrm{TM}}$ (BD Biosciences). The cells were seeded at a density of $1 \times 10^{5}$ cells on the upper chamber with $200 \mu \mathrm{l}$ serum-free DMEM. Following transfection for $48 \mathrm{~h}$, $600 \mu 1$ DMEM supplemented with $20 \%$ FBS, which served as a chemoattractant, was added to the lower chamber. After $48 \mathrm{~h}$ incubation at $37^{\circ} \mathrm{C}$, the upper side of the membrane was wiped with cotton wool to remove noninvasive cells; the membranes were then fixed with $4 \%$ methanol at $4^{\circ} \mathrm{C}$ and stained with $0.1 \%$ crystal violet at room temperature. Five visual fields with a magnification $\mathrm{x} 200$ were randomly selected from each membrane and the cell numbers were counted using a light microscope.

Statistical analysis. All data are expressed as the mean \pm standard deviation $(n=3)$. The significance of differences between two groups was estimated using a Student's t-test. The significance of differences between multiple groups was determines 


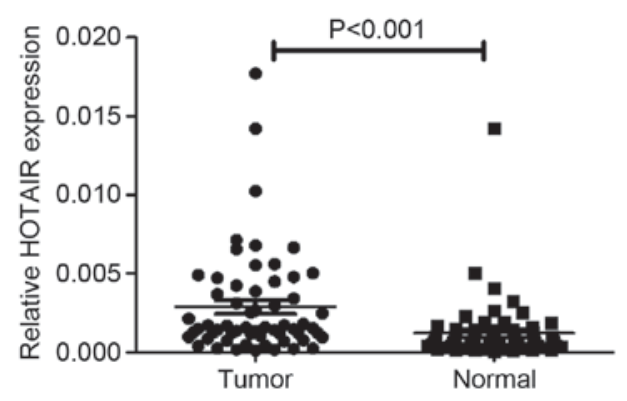

Figure 1. HOTAIR expression is upregulated in OS tissues. The relative expression of HOTAIR in OS tissues and adjacent normal tissues was analyzed using reverse transcription-quantitative polymerase chain reaction analysis and normalized to GAPDH expression. HOTAIR, Hox transcript antisense intergenic RNA; OS, osteosarcoma.

using one-way analysis of variance followed by a post hoc Tukey's range test. SPSS software (version 21.0; IBM Corp., Armonk, NY, USA) was used to perform the statistical analysis. All tests performed were two sided. $\mathrm{P}<0.05$ was considered to indicate a statistically significant difference.

\section{Results}

HOTAIR expression is significantly increased and correlated with lymph node metastasis in patients with OS. RT-qPCR analysis determined that the expression of HOTAIR was significantly increased in clinical OS specimens compared with adjacent normal tissues ( $\mathrm{P}<0.001$; Fig. 1). Clinicopathological analysis demonstrated that when the 60 tissue pairs were tested, the expression of HOTAIR demonstrated no difference between age, gender or tumor size; however, it was significantly associated with tumor grade $(\mathrm{P}=0.035)$ and distant metastasis $(\mathrm{P}=0.007)$ (Table I). These results indicate that HOTAIR serves an oncogenic role in OS.

HOTAIR silencing reduces the proliferation of MG-63 cells. To determine whether HOTAIR affects tumorigenesis, MG-63 cell lines were transfected with siR-HOTAIR1/2 or siR-NC.
RT-qPCR results revealed that, after being transfected with siR-HOTAIR1/2, HOTAIR mRNA expression in the MG-63 cells decreased significantly when compared to the mock group ( $\mathrm{P}<0.01$; Fig. 2A). A CCK-8 assay demonstrated that when HOTAIR was knocked down, the survival rate of MG-63 cells was significantly inhibited $(\mathrm{P}<0.05$; Fig. $2 \mathrm{~B})$. This data supports the hypothesis that HOTAIR serves a role in OS cell proliferation.

HOTAIR silencing induces the $G_{1}$ phase cell cycle arrest of $M G-63$ cells. Flow cytometry analysis was performed to determine whether the reduced expression of HOTAIR contributed to cell cycle arrest or apoptosis. It was revealed that MG-63 cells of siR-HOTAIR1 group and siR-HOTAIR2 group had a significant increase in the proportion of cells in the $\mathrm{G}_{1}$ phase compared with the mock group ( $\mathrm{P}<0.05$; Fig. $\left.3 \mathrm{~A}\right)$. This suggests that the cells have been prevented from moving into the next phase of the cell cycle and have therefore been prevented from proliferating. It was also revealed that the reduction in HOTAIR expression did not significantly influence the apoptosis of MG-63 cells (Fig. 3B). This data suggests that the inhibitory effect HOTAIR silencing has on the proliferation of MG-63 cells is through cell cycle arrest and not apoptosis.

Knockdown of HOTAIR inhibits the migration and invasion of $M G-63$ cells. To assess the effects of HOTAIR on cell migration and invasion, a Transwell migration assay was performed. MG-63 cells transfected with siR-HOTAIR1 or siR-HOTAIR2 exhibited a significant reduction in migration compared to the mock group ( $\mathrm{P}<0.01$; Fig. $4 \mathrm{~A}$ and $\mathrm{B})$. A Boyden chamber coated with Matrigel was used to determine the effect of siR-HOTAIR on cell invasion after incubation for $48 \mathrm{~h}$. The results revealed that HOTAIR silencing significantly decreased the invasion of MG-63 cells $(\mathrm{P}<0.01$; Fig. 4A and $\mathrm{C})$. The suppressive effects of siR-HOTAIR explain the association observed between high HOTAIR expression and distant metastasis in patients with OS. This data indicates that siR-HOTAIR can inhibit a migratory and invasive phenotype in OS cells.
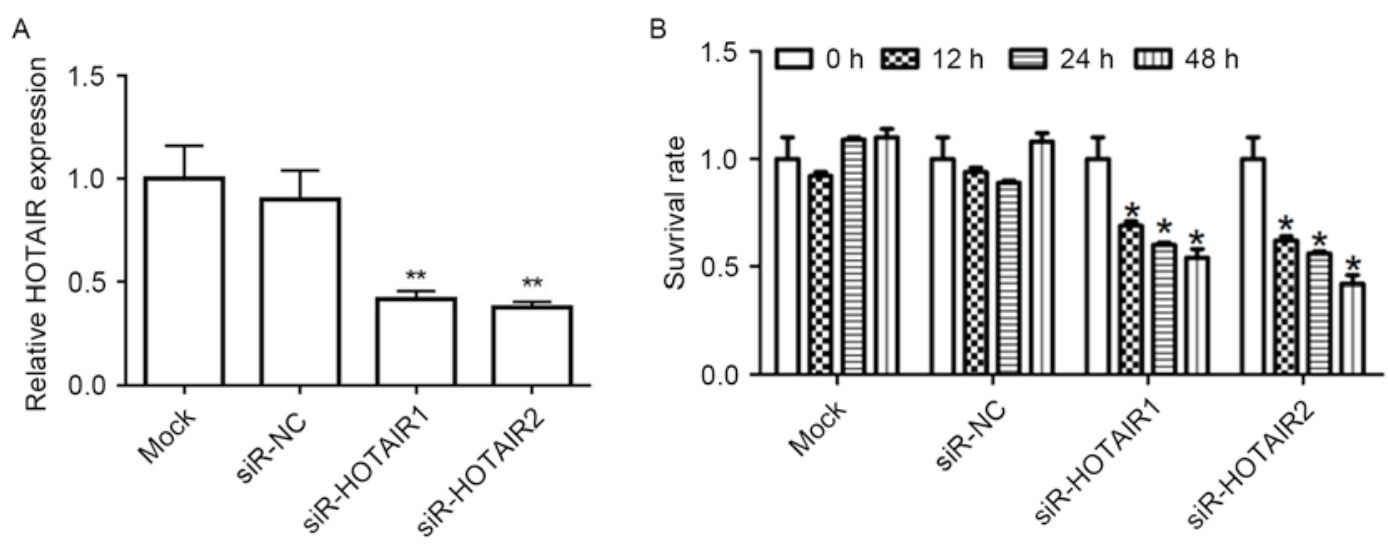

Figure 2. HOTAIR silencing inhibits the proliferation of MG-63 cells. (A) MG-63 cells were transfected with siR-HOTAIR, after 48 h the relative expression of HOTAIR was measured by reverse transcription-quantitative polymerase chain reaction analysis. (B) MG-63 cells were transfected with siR-HOTAIR or siR-NC for 12,24 and $48 \mathrm{~h}$, then cell viability was measured using the Cell Counting Kit- 8 assay. ${ }^{* *} \mathrm{P}<0.01$, ${ }^{*} \mathrm{P}<0.05$ vs. the mock group. HOTAIR, Hox transcript antisense intergenic RNA; siR, small interfering RNA; NC, negative control. 

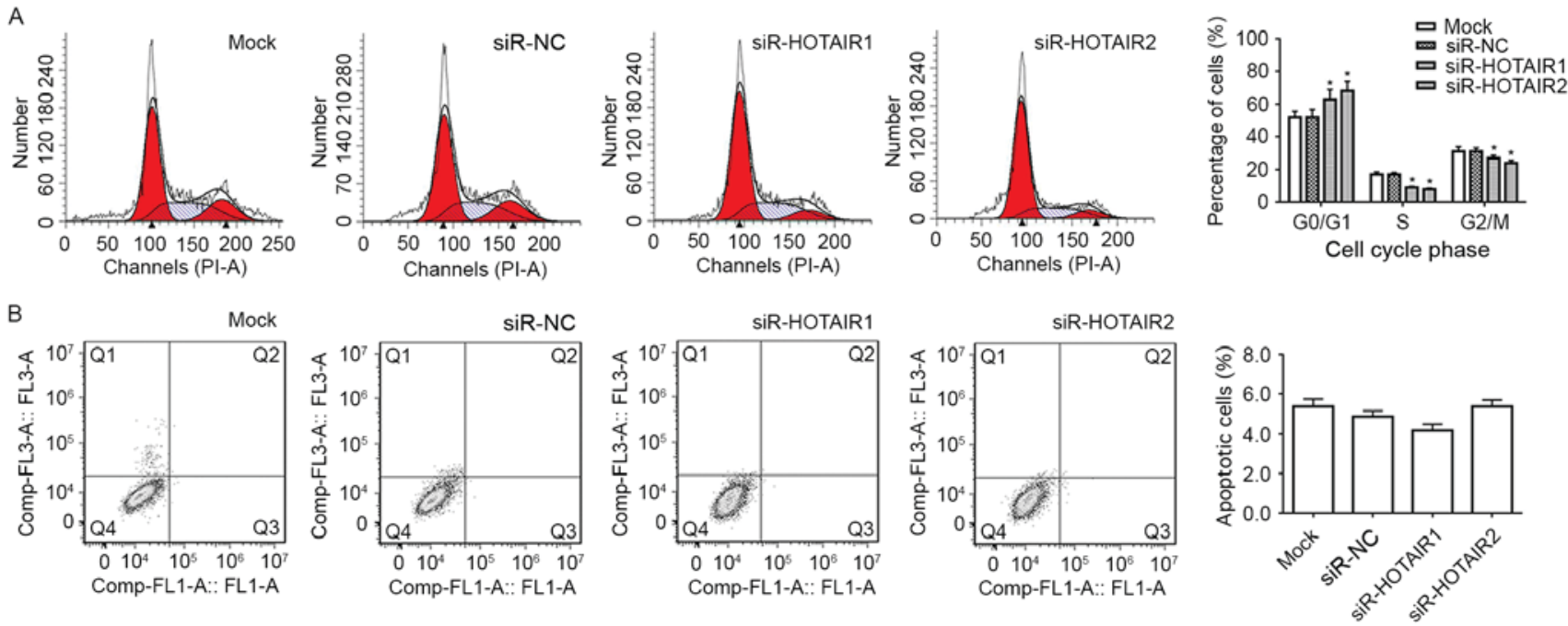

Figure 3. Downregulation of HOTAIR expression arrests the cell cycle at the $\mathrm{G}_{1}$ phase. MG-63 cells were treated with siR-HOTAIR or siR-NC. (A) Flow cytometry analysis revealed a significant increase in the proportion of cells in the $\mathrm{G}_{1}$ phase in the siR-HOTAIR group compared with the mock group. (B) Flow cytometry analysis also demonstrated that downregulation of HOTAIR expression did not significantly affect the apoptosis of MG- 63 cells. ${ }^{*} \mathrm{P}<0.05$ vs. the mock group. HOTAIR, Hox transcript antisense intergenic RNA; siR, small interfering RNA; NC, negative control.

A
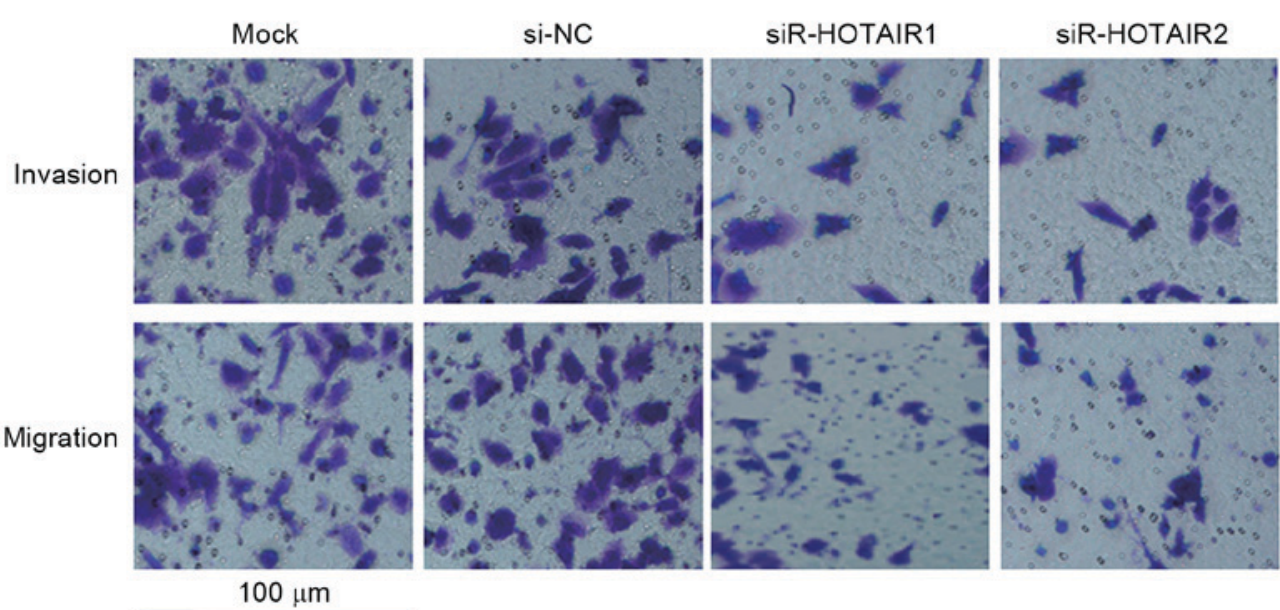

B

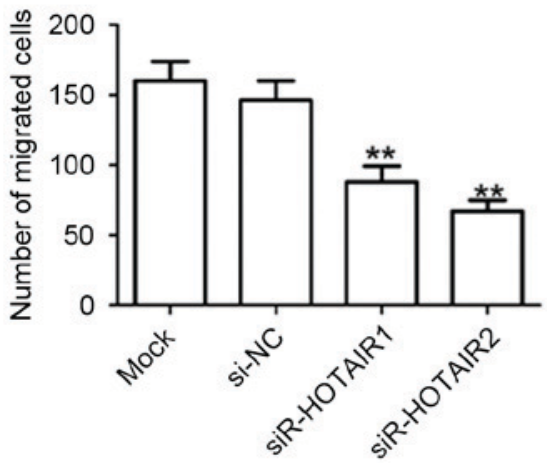

C
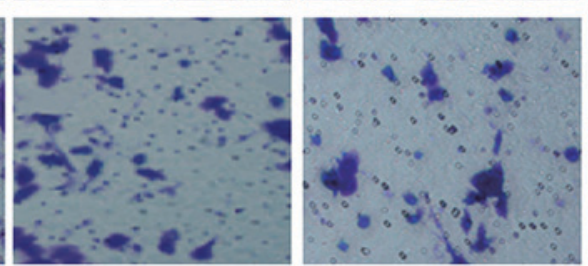
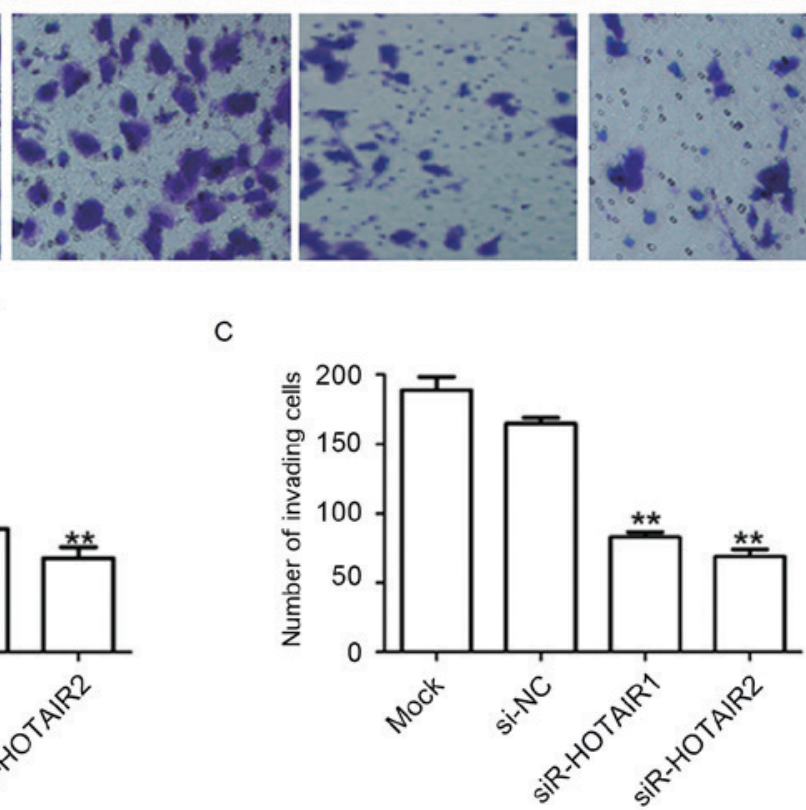

Figure 4. HOTAIR silencing significantly reduces the migration and invasion of MG-63 cells. (A) MG-63 cells were transfected with siR-HOTAIR or siR-NC for $48 \mathrm{~h}$ and images were taken using a light microscope. Magnification, x200. A Transwell assay was performed for $48 \mathrm{~h}$, and the number of (B) migrating cells and $(\mathrm{C})$ invading cells were calculated. ${ }^{* *} \mathrm{P}<0.01$ vs. the mock group. HOTAIR, Hox transcript antisense intergenic RNA; siR, small interfering RNA; NC, negative control.

HOTAIR regulates the expression of cyclin D1, cyclin E, CDK2, CDK4 and 27 within OS cells. To investigate the evidence that siR-HOTAIR serves a role in $\mathrm{G}_{1} / \mathrm{S}$ arrest, RT-qPCR (Fig. 5A) and western blot analysis (Fig. 5B) were used to evaluate the effect of HOTAIR silencing on the expression of CDK2, CDK4, cyclin D1 and cyclin E, all of which are associated with progression from the $G_{1}$ to $S$ phase in the cell cycle. The CDK inhibitor p27 was also studied. The 
A

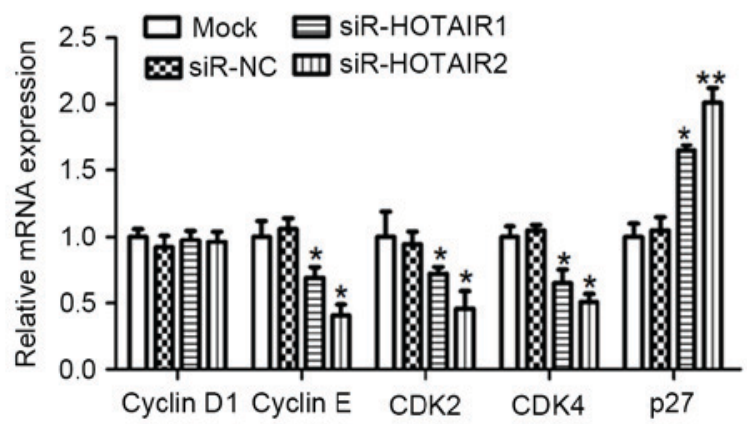

B

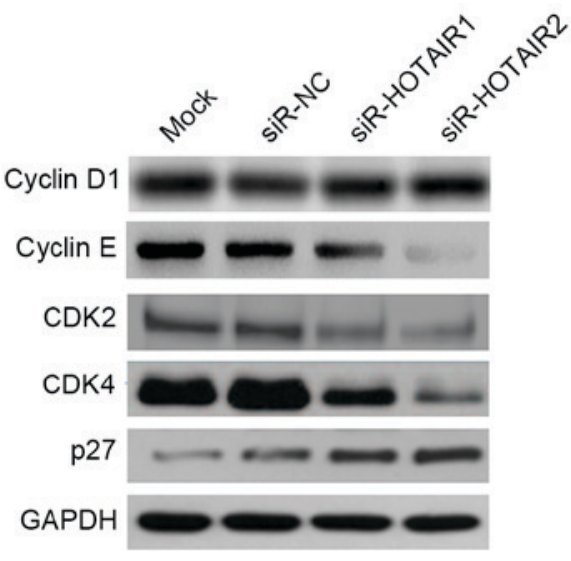

Figure 5. HOTAIR silencing affects the expression of growth-associated proteins in MG-63 cells. The mRNA and protein expression of cyclin D1, cyclin E, CDK2, CDK4 and p27 were detected by (A) reverse transcription-quantitative polymerase chain reaction and (B) western blot analysis. ${ }^{*} \mathrm{P}<0.05,{ }^{* *} \mathrm{P}<0.01 \mathrm{vs}$. the mock group. HOTAIR, Hox transcript antisense intergenic RNA; siR, small interfering RNA; NC, negative control. Cyclin D1, G/ $/ S$-specific cyclin D1; CDK, cyclin-dependent kinase.

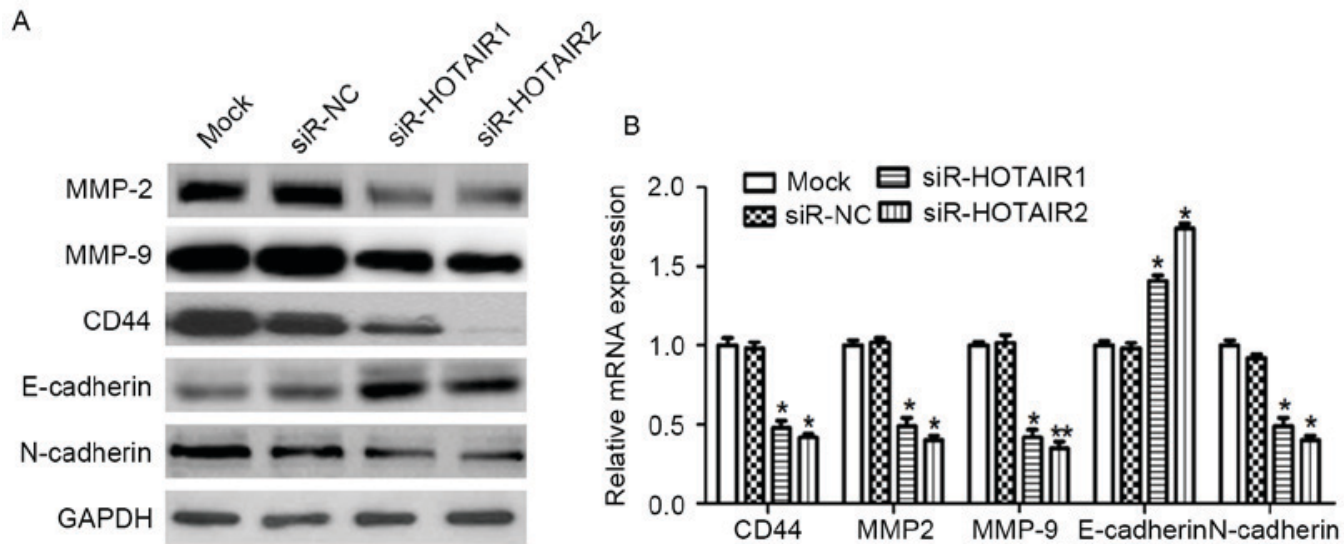

Figure 6. HOTAIR silencing alters the expression of proteins associated with tumor invasion and metastasis in MG-63 cells. The protein and mRNA expression of MMP2, MMP9, CD44, E-cadherin and N-cadherin were detected by (A) western blot analysis and (B) reverse transcription-quantitative polymerase chain reaction analysis. "P $<0.05,{ }^{* *} \mathrm{P}<0.01$ vs. the mock group. HOTAIR, Hox transcript antisense intergenic RNA; siR, small interfering RNA; NC, negative control. MMP, matrix metalloproteinase; E-cadherin, epithelial-cadherin; CD, cluster of differentiation.

results demonstrated that HOTAIR silencing significantly reduced the mRNA expression of CDK2, CDK4 and cyclin $\mathrm{E}$ compared to the controls $(\mathrm{P}<0.01)$, while cyclin $\mathrm{D} 1$ exhibited little variation. Conversely, the mRNA expression of p27 was significantly increased compared to the control $(\mathrm{P}<0.05)$. Similar results were observed for protein expression. These results suggest that HOTAIR mediates OS cell proliferation by affecting cyclin E, CDK2, CDK4 and p27 expression.

Expression of MMP2, MMP9, CD44, E-cadherin and $N$-cadherin is involved in the HOTAIR-mediated promotion of OS cell metastasis. To explore the molecular mechanisms underlying the effect of HOTAIR on the invasion and metastasis of OS cells, potential targets involved in tumor invasion and metastasis were investigated. The results revealed a marked decrease in CD44, MMP2, MMP9 and N-cadherin expression, and a notable increase in E-cadherin expression at the protein level (Fig. 6A). At the mRNA level a similar trend was observed, which was significant in the siR-HOTAIR groups compared to the mock group $(\mathrm{P}<0.01$; Fig. 6B). This data suggests an association between HOTAIR and MMP2, MMP9, CD44, E-cadherin and N-cadherin expression, as HOTAIR treatment resulted in increased expression levels of MMP2, MMP9, CD44 and N-cadherin, but decreased expression levels of E-cadherin, which indicates that these factors may serve an important role in the HOTAIR-mediated promotion of OS cell invasion and metastasis.

HOTAIR promotes OS cell growth through activation of the AKT/mTOR signaling pathway. The AKT/mTOR signaling pathway serves a key role in regulating cell growth, survival and metabolism. To further explore the molecular mechanisms by which HOTAIR affects OS cell proliferation and metastasis, the effects of HOTAIR silencing on the AKT/mTOR signaling pathway were investigated. In the present study the expression of three key proteins, p-mTOR (ser2448), p-PI3K (tyr458) and p-AKT (ser473), was detected. HOTAIR silencing reduced the expression of p-mTOR, p-PI3K and p-AKT, while no notable changes were observed in the unphosphorylated proteins (Fig. 7). These results suggest that HOTAIR promotes 


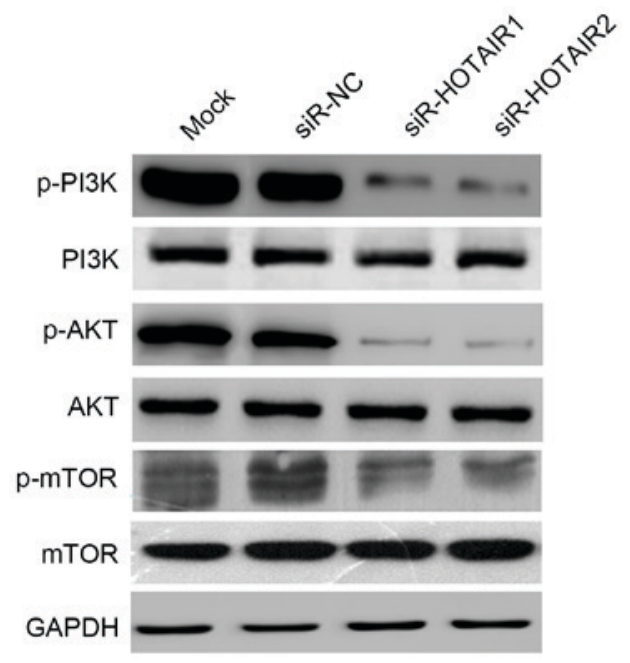

Figure 7. Silencing HOTAIR decreases the phosphorylation of AKT/mTOR signaling pathway proteins. The expression of AKT/mTOR signaling pathway proteins, including p-mTOR (ser2448), p-PI3K (tyr458) and p-AKT, and their unphosphorylated counterparts, was examined by western blot analysis. HOTAIR, Hox transcript antisense intergenic RNA; AKT, RAC a serine/threonine-protein kinase; mTOR, mammalian target of rapamycin; p, phosphorylated; PI3K, phosphoinositide 3-kinase.

OS cell growth through activation of the AKT/mTOR signaling pathway.

\section{Discussion}

Numerous studies have demonstrated that changes in the expression of certain lncRNAs are closely associated to the occurrence and development of OS tumors $(18,19)$. It has also been revealed that IncRNAs serve an important role in the invasion and metastasis of OS cells $(20,21)$. These lncRNAs can act as oncogenes or tumor suppressors $(3,4)$. Therefore, an improved understanding of the aberrant expression of lncRNAs within OS cells may help to clarify the underlying mechanisms of OS metastasis and provide novel targets for therapies.

Recent studies have revealed that the IncRNA HOTAIR is an oncogene in several different types of cancer, including breast cancer, bladder cancer, colorectal cancer and OS (22-24). In the present study, it was revealed that HOTAIR was upregulated in clinical OS tissue samples, indicating that HOTAIR is a potential molecular marker for OS. The present study used siRNA technology to effectively knockdown HOTAIR mRNA expression in human MG-63 OS cells. It was hypothesized that HOTAIR silencing may suppress OS cell proliferation, adhesion, migration and invasion ability, and thus it may serve a tumor suppressor role within OS cells.

Cyclin D1/E and CDK2/4 are considered important tumor proliferation-associated genes $(25,26)$. To determine the molecular mechanism by which HOTAIR affects the growth of OS cells, the effect of HOTAIR silencing on the regulation of the cell cycle was examined. Cyclin $E$ binds to $G_{1}$ phase CDK2 to form a complex, which is required for the transition from the $G_{1}$ to $S$ phase of the cell cycle (27). Cyclin D1 forms a complex with and functions as a regulatory subunit of CDK4, whose activity is also required for $\mathrm{G}_{1} / \mathrm{S}$ transition (25). In the present study it was revealed that HOTAIR silencing reduced Cyclin E, but not Cyclin D1 expression. p27 binds to and prevents the activation of cyclin E-CDK2 and cyclin D-CDK4 complexes, and thus controls the cell cycle progression at the G1 stage (26). HOTAIR silencing resulted in the upregulation of $\mathrm{p} 27$. These results indicate that HOTAIR decreases the expression of $\mathrm{p} 27$, reducing control over the activation of cyclin E/CDK2 and cyclin D-CDK4 complexes, resulting in dysregulation of the cell cycle and thus the proliferation of OS cells.

Tumor metastasis and invasion are closely associated to the degradation of a variety of metastasis-associated proteins and the destruction of the extracellular matrix (ECM). MMP-2/9, CD44, E-cadherin and N-cadherin are all important tumor metastasis-associated proteins. MMPs are considered the most important set of proteases that degrade the ECM. MMP2 and MMP9 are the most thoroughly studied MMPs (28). CD44 is a cell-surface glycoprotein involved in cell-cell interactions, cell adhesion and migration. CD44 is a receptor for hyaluronic acid and can also interact with other ligands, including osteopontin, collagens and MMPs (29). E-cadherin, whose function is critical for cell-cell adhesion and developmental morphogenesis, serves a key role in cellular invasion. The loss of E-cadherin contributes to the movement of tumor cells from their primary location. Often tumors that have an increased degree of malignancy also have an increased degree of invasion. E-cadherin can reduce the invasion ability of a number of types of tumor cells (30). N-cadherin is a transmembrane protein expressed in multiple tissues, which can provide a mechanism for the transendothelial migration of cancer cells. If the proto-oncogene tyrosine-protein kinase Src (Src) signaling pathway is upregulated, Src phosphorylates $\beta$-catenins attached to $\mathrm{N}$-cadherin and E-cadherin, which can lead to the failure of the intercellular connection, allowing the cancer cells to metastasize (31).

The AKT/mTOR signaling pathway is a tumor-associated signaling pathway. Activated AKT is known to regulate downstream signaling proteins involved in cell survival, cell growth, cell cycle progression (32). and apoptosis (26). Overexpression of p-AKT is associated with advanced human prostate cancer (33). mTOR, a protein downstream of AKT, also serves a vital role in regulating cell proliferation, growth, differentiation and survival. The present study revealed that the silencing of HOTAIR expression notably reduced the phosphorylation of mTOR and its upstream kinase AKT, suggesting that HOTAIR silencing suppresses tumorigenesis by inhibiting the AKT/mTOR signaling pathway and its multiple downstream signaling components in OS cells.

In conclusion, the results of the present study provide evidence that the expression of the IncRNA HOTAIR is increased in OS and that it is associated with the progression of OS. HOTAIR silencing inhibited the growth, adhesion, migration and invasion of MG-63 OS cells. Therefore, HOTAIR silencing may potentially form the basis of novel cancer treatments.

\section{References}

1. Cortini M, Avnet S and Baldini N: Mesenchymal stroma: Role in osteosarcoma progression. Cancer Lett 405: 90-99, 2017. 
2. Yu W, Tang L, Lin F, Yao Y, Shen Z and Zhou X: The role of chemotherapy for metastatic, relapsed and refractory osteosarcoma. Surg Oncol 1: 9-15, 2015.

3. Bartonicek N, Maag JL and Dinger ME: Long noncoding RNAs in cancer: Mechanisms of action and technological advancements. Mol Cancer 15: 43, 2016.

4. Lavorgna G, Vago R, Sarmini M, Montorsi F, Salonia A and Bellone M: Long non-coding RNAs as novel therapeutic targets in cancer. Pharmacol Res 110: 131-138, 2016.

5. Wang CF and Zhu XZ: The fourth edition of WHO classification of tumours of bone: An introduction. Zhonghua Bing Li Xue Za Zhi 10: 652-654, 2013 (In Chinese).

6. Xue M, Li X, Wu W, Zhang S, Wu S Li Z and Chen W: Upregulation of long non-coding RNA urothelial carcinoma associated 1 by CCAAT/enhancer binding protein $\alpha$ contributes to bladder cancer cell growth and reduced apoptosis. Oncol Rep 5: 1993-2000, 2014.

7. Yang MH, Hu ZY, Xu C, Xie LY, Wang XY, Chen SY and Li ZG: MALAT1 promotes colorectal cancer cell proliferation/migration/invasion via PRKA kinase anchor protein 9. Biochim Biophys Acta 1852: 166-174, 2015.

8. Li J, Wang J, Chen Y, Li S, Jin M, Wang H, Chen Z and Yu W: LncRNA MALAT1 exerts oncogenic functions in lung adenocarcinoma by targeting miR-204. Am J Cancer Res 6: 1099-1107, 2016.

9. Miao Y, Fan R, Chen L and Qian H: Clinical significance of long Non-coding RNA MALAT1 expression in tissue and serum of breast cancer. Ann Clin Lab Sci 46: 418-424, 2016.

10. Zhou Y, Xu X, Lv H, Wen Q, Li J, Tan L, Li J and Sheng X: The long noncoding RNA MALAT-1 is highly expressed in ovarian cancer and induces cell growth and migration. PLoS One 11: e0155250, 2016.

11. Lai MC, Yang Z, Zhou L, Zhu QQ, Xie HY, Zhang F, Wu LM, Chen LM and Zheng SS: Long non-coding RNA MALAT-1 overexpression predicts tumor recurrence of hepatocellular carcinoma after liver transplantation. Med Oncol 29: 1810-1816, 2012.

12. Cai B, Song XQ, Cai JP and Zhang S: HOTAIR: A cancer-related long non-coding RNA. Neoplasma 64: 379-391, 2014.

13. Wu Y, Zhang L, Zhang L, Wang Y, Li H, Ren X, Wei F, Yu W, Liu T, Wang X, et al: Long non-coding RNA HOTAIR promotes tumor cell invasion and metastasis by recruiting EZH2 and repressing E-cadherin in oral squamous cell carcinoma. Int J Oncol 46: 2586-2594, 2015.

14. Lee NK, Lee JH, Park CH, Yu D, Lee YC, Cheong JH, Noh SH and Lee SK: Long non-coding RNA HOTAIR promotes carcinogenesis and invasion of gastric adenocarcinoma. Biochem Biophys Res Commun 45: 171-178, 2014.

15. Gupta RA, Shah N, Wang KC, Kim J, Horlings HM, Wong DJ, Tsai MC, Hung T, Argani P, Rinn JL, et al: Long non-coding RNA HOTAIR reprograms chromatin state to promote cancer metastasis. Nature 464: 1071-1076, 2010.

16. Zhang Z, Cheng J, Wu Y, Qiu J, Sun Y and Tong X: LncRNA HOTAIR controls the expression of Rab22a by sponging miR-373 in ovarian cancer. Mol Med Rep 14: 2465-2472, 2016.

17. Livak KJ and Schmittgen TD: Analysis of relative gene expression data using real-time quantitative PCR and the 2(-Delta Delta C(T)) method. Methods 25: 402-408, 2001
18. Wang G, Cui T, Sun L, Peng N and Yang C: Long noncoding RNA LeXis promotes osteosarcoma growth through upregulation of CTNNB1 expression. Am J Cancer Res 7: 1577-1587, 2017.

19. Li Z, Dou P, Liu T and He S: Application of long noncoding RNAs in osteosarcoma: Biomarkers and therapeutic targets. Cell Physiol Biochem 42: 1407-1419, 2017.

20. Li Z, Zhao L and Wang Q: Overexpression of long non-coding RNA HOTTIP increases chemoresistance of osteosarcoma cell by activating the Wnt/ $\beta$-catenin pathway. Am J Transl Res 8: 2385-2393, 2016

21. Li W, Xie P and Ruan WH: Overexpression of lncRNA UCA1 promotes osteosarcoma progression and correlates with poor prognosis. J Bone Oncol 5: 80-85, 2016

22. Xue X, Yang YA, Zhang A, Fong KW, Kim J, Song B, Li S, Zhao JC and Yu J: LncRNA HOTAIR enhances ER signaling and confers tamoxifen resistance in breast cancer. Oncogene 35: 2746-2755, 2016.

23. Sun X, Du P, Yuan W, Du Z, Yu M, Yu X and Hu T: Long non-coding RNA HOTAIR regulates cyclin $\mathrm{J}$ via inhibition of microRNA-205 expression in bladder cancer. Cell Death Dis 6: e1907, 2015.

24. Xue Y, Gu D, Ma G, Zhu L, Hua Q, Chu H, Tong N, Chen J, Zhang $\mathrm{Z}$ and Wang M: Genetic variants in lncRNA HOTAIR are associated with risk of colorectal cancer. Mutagenesis 30: 303-310, 2015.

25. Wang B, Su Y, Yang Q, Lv D, Zhang W, Tang K, Wang H, Zhang R and Liu Y: Overexpression of long Non-coding RNA HOTAIR promotes tumor growth and metastasis in human osteosarcoma. Mol Cells 38: 432-440, 2015.

26. Takahashi-Yanaga $\mathrm{F}$ and Sasaguri T: GSK-3beta regulates cyclin D1 expression: A new target for chemotherapy. Cell Signal 20: 581-589, 2008.

27. Sheaff RJ, Groudine M, Gordon M, Roberts JM and Clurman BE: Cyclin E-CDK2 is a regulator of p27Kip1. Genes Dev 11: 1464-1178, 1997.

28. Yue J, Zhang K and Chen J: Role of integrins in regulating proteases to mediate extracellular matrix remodeling. Cancer Microenviron 5: 275-283, 2012

29. Yu Q and Stamenkovic I: Localization of matrix metalloproteinase 9 to the cell surface provides a mechanism for CD44-mediated tumor invasion. Genes Dev 13: 35-48, 1999.

30. Canel M, Serrels A, Frame MC and Brunton VG: E-cadherin-integrin crosstalk in cancer invasion and metastasis. J Cell Sci 126: 393-401, 2013.

31. Yang P, Qiu Z, Jiang Y, Dong L, Yang W, Gu C, Li G and Zhu Y: Silencing of cZNF292 circular RNA suppresses human glioma tube formation via the Wnt/ $\beta$-catenin signaling pathway. Oncotarget 7: 63449-63455, 2016.

32. Sokolowski KM, Koprowski S, Kunnimalaiyaan S, Balamurugan M, Gamblin TC and Kunnimalaiyaan M: Potential molecular targeted therapeutics: Role of PI3-K/AKT/mTOR inhibition in cancer. Anticancer Agents Med Chem 16: 29-37, 2016.

33. Tan J, Jiang X, Yin G, He L, Liu J, Long Z, Jiang Z and Yao K: Anacardic acid induces cell apoptosis of prostatic cancer through autophagy by ER stress/DAPK3/Akt signaling pathway. Oncol Rep 38: 1373-1382, 2017. 